\title{
Revertant fibres and dystrophin traces in Duchenne muscular dystrophy: implication for clinical trials.
}

\author{
Virginia Arechavala-Gomeza ${ }^{1}$, Maria Kinali ${ }^{1,2}$, L Feng ${ }^{1}$, Michela Guglieri ${ }^{3}$, Geraldine \\ Edge $^{4}$, Marion Main ${ }^{1}$, David Hunt ${ }^{5}$, Jan Lehovsky ${ }^{6}$ Volker Straub ${ }^{3}$, Kate Bushby ${ }^{3}$, \\ Caroline Sewry ${ }^{1,7}$, Jennifer Morgan ${ }^{1}$, Francesco Muntoni ${ }^{1}$.
}

\footnotetext{
${ }^{1}$ The Dubowitz Neuromuscular Centre, Institute of Child Health, University College London, ${ }^{2}$ Department of Pediatrics, Hammersmith Hospital, London' ${ }^{3}$ Institute of Human Genetics, Newcastle University, Newcastle, UK ${ }^{4}$ Department of Anaesthetics, Stanmore Hospital, London' ${ }^{5}$ Department of Orthopaedics, St. Mary's Hospital, London, ${ }^{6}$ Department of Orthopaedics, Stanmore Hospital, London, ${ }^{7}$ Centre for Inherited Neuromuscular Diseases, RJAH Orthopaedic Hospital, Oswestry
}

\section{Abstract}

Duchenne Muscular Dystrophy (DMD) is characterised by the absence of dystrophin in muscle biopsies, although residual dystrophin can be present, either as dystrophin positive (revertant) fibres or traces. As restoration of dystrophin expression is the end point of clinical trials, such residual dystrophin is a key factor in recruitment of patients and may also confound the analysis of dystrophin restoration in treated patients, if, as previously observed in the $m d x$ mouse, revertant fibres increase with age. In $62 \%$ of the diagnostic biopsies reports of 65 DMD patients studied, traces or revertants were recorded with no correlation between traces or revertants, the patients' performance, or corticosteroids response. In nine of these patients, there was no increase in traces or revertants in biopsies taken a mean of 8.23 years (5.8-10.4 years) after the original diagnostic biopsy. This information should help in the design and execution of clinical trials focused on dystrophin restoration strategies.

Keywords: Duchenne Muscular Dystrophy ; Revertant fibres ; Dystrophin expression

\section{Introduction}

Duchenne muscular dystrophy (DMD) is caused by mutations in the $D M D$ gene that prevent the production of functional dystrophin. These mutations (both large rearrangements or small mutations) cause a disruption of the open reading frame (ORF) impeding the correct protein translation in DMD patients [1]. In the milder Becker muscular dystrophy (BMD) intragenic mutations that maintain the dystrophin mRNA ORF give rise to internally-deleted but partially functional dystrophins [1,2].

The diagnosis of DMD is corroborated by the demonstration of absent dystrophin. In muscle biopsy sections immunolabelled with antibodies to dystrophin, this is reflected as a dystrophin-negative background [3]. However, very rare, strikingly dystrophin-positive fibres [4] observed singly or in clusters, have been described in approximately $50 \%$ of DMD cases
[1,4-10]. These "revertant fibres" [11] are also present in the $m d x$ mouse [4] and the golden retriever dystrophic dog (GRMD) animal models [12]. The percentage of revertant fibres reported in diagnostic biopsies from DMD patients ranges from 0.01 to $7 \%$ [79,11,13-15] while in the $m d x$ mouse they account for less than $1 \%$ of all muscle fibres $[4,16]$. A less reported though well known phenomenon [17] is the presence of traces of dystrophin, described as patches of belownormal dystrophin-positive areas visible at the sarcolemma of muscle fibres, seen in approximately $20 \%$ of DMD patients $[6,17]$. Traces may be present in up to $25 \%$ of the fibres [17] in DMD patients but do not appear to occur in the $m d x$ mouse [18]. It is not known if these traces of dystrophin have a functional effect. [13] The extent and coexistence of revertant fibres and dystrophin

Correspondence to: Jennifer Morgan, The Dubowitz Neuromuscular Centre, $1^{\text {st }}$ Floor, UCL Institute of Child Health, 30 Guilford Street, London, WC1N EH Tel: +44 (0)20 7905 2874, Fax: +44 (0)20 7905 2832;

j.morgan@ich.ucl.ac.uk 
traces have not been well characterized in DMD boys.

Revertant fibres have internally-deleted dystrophins, which skip frame-shifting normal exons inducing favourable skipping events and restoring the ORF in DMD patients [19]. These revertant dystrophins often lack the exon domains flanking deletions $[11,13,20]$ and in the $m d x$ mouse the mutated exon 23 [19] but conserve the $\mathrm{N}$ and $\mathrm{C}$ terminal domains [11] allowing them to be correctly localized to the sarcolemma [21]. Studies in both DMD patients [14,22] and the $m d x$ mouse $[18,19,23]$ have shown that an individual may harbor several types of revertant fibres, consisting of different internally deleted dystrophins. Proposed mechanisms of their generation include somatic reversion [24] and alternative splicing $[9,19]$, the latter described in the coding region of the DMD gene in both patients and unaffected individuals [20]. As an endpoint in several ongoing clinical trials in DMD is the restoration of dystrophin expression[25-27], the interpretation of preexisting residual dystrophin expression (both revertant fibres and trace expression) is important when assessing the efficiency of the therapeutic intervention. If revertant fibres increased in number with age, this may confound the interpretation of the intervention, especially in studies lasting several years and in which the increase of dystrophin is only limited.

Our study provides essential information on the residual dystrophin expression in a relatively large population of DMD patients. We firstly performed a detailed analysis of the previous diagnostic biopsy reports to assess the frequency of revertant fibres and dystrophin traces, and correlated the dystrophin expression findings to the severity of the disease progression. Finally, in a subset of patients we studied the presence of traces and revertant fibres over time in different muscles (original diagnostic quadriceps compared with extensor digitorum brevis, EDB, or paraspinal muscles).

\section{Materials and Methods DMD patients}

All patients in this study had been diagnosed and followed up at the Dubowitz Neuromuscular Centre at Hammersmith Hospital, London, recently relocated to the Institute of Child Health \& Great Ormond Street Hospital for Children, London, and at the Institute of Human Genetics in Newcastle, both tertiary referral centres for children with neuromuscular disorders. All patients presented with a DMD clinical picture, confirmed by an elevated serum CK, a DMD gene deletion and absence of dystrophin from the majority of fibres in a muscle biopsy [28].

Patients had detailed assessments of muscle strength and functional abilities using the Hammersmith Motor Ability Score (MAS) (maximum score 40) [29]. MAS deteriorates with advancing age and stabilizes or improves with steroid treatment $[29,30]$. We collected data on the MAS at baseline and within the first 3-6 months, i.e. at the first clinic review following the initiation of prednisolone therapy. We correlated the presence of residual dystrophin expression with the best MAS and the patient's age prior to any intervention including the onset of prednisolone.

\section{DMD muscle biopsies}

Diagnostic biopsies (needle or open) were obtained from quadriceps muscles. In a subset of nine wheelchair-dependent patients (mean age 11.9 years), the extensor digitorum brevis (EDB) muscles were collected several years after (mean 8.23 years) their diagnostic biopsies during surgery for an orthopaedic procedure (Supplementary table 1).

Muscle biopsy specimens were mounted in OCT, frozen by immersion in isopentane cooled in liquid nitrogen and stored at $-80^{\circ} \mathrm{C}$. Control sections were taken from a thigh muscle biopsy of a 41-year old female, with minimal myopathic changes. All muscle biopsies were processed by the same pathology team. 


\begin{tabular}{|c|c|c|c|c|c|c|c|}
\hline \multirow[t]{2}{*}{ Patient } & \multirow[t]{2}{*}{ Mutation } & \multicolumn{2}{|c|}{ Diagnostic biopsy } & \multicolumn{2}{|r|}{ EDB biopsy } & \multirow{2}{*}{$\begin{array}{c}\text { Time } \\
\text { between } \\
\text { biopsies } \\
\text { (years) }\end{array}$} & \multirow{2}{*}{$\begin{array}{l}\text { Increase in } \\
\text { revertants? }\end{array}$} \\
\hline & & Age & Report & Age & Report & & \\
\hline A & Mutation exon 70 & 3 & No revertants & 13 & $\begin{array}{l}\text { Right EDB No } \\
\text { revertants }\end{array}$ & 10.4 & No \\
\hline $\mathbf{A b}$ & Mutation exon 70 & 3 & “ & 13 & $\begin{array}{l}\text { Left EDB No } \\
\text { revertants }\end{array}$ & 10.4 & No \\
\hline B & Del exons 46-52 & 5 & $\begin{array}{l}\text { Revertant } \\
\text { fibres }\end{array}$ & 12 & Revertant fibres & 7.9 & No \\
\hline C & Del exons 3-13 & 4 & Traces & 11 & Traces & 6.9 & No \\
\hline D & Del exons 44 & 3 & $\begin{array}{l}\text { Revertant } \\
\text { fibres }\end{array}$ & 10 & Revertant fibres & 6.4 & No \\
\hline E & Del exons 50-53 & 2 & No revertants & 8 & One revertant & 6 & No* \\
\hline $\mathbf{F}$ & Del exons 45-52 & 4 & Faint traces & 11 & $\begin{array}{l}\text { Occasional } \\
\text { traces }\end{array}$ & 7 & No \\
\hline G & Del exons 46-51 & 5.6 & $\begin{array}{l}\text { Revertant } \\
\text { fibres }\end{array}$ & 12 & Revertant fibres & 6.7 & No \\
\hline H & Del exon 50 & 8 & $\begin{array}{l}\text { Traces and } \\
\text { revertants }\end{array}$ & 16 & $\begin{array}{l}\text { Traces and } \\
\text { revertants }\end{array}$ & 8.8 & No \\
\hline I & Del exon 50 & 7 & $\begin{array}{c}\text { No revertants, } \\
\text { faint traces }\end{array}$ & 13 & $\begin{array}{l}\text { Some revertants } \\
\text { and faint traces }\end{array}$ & 5.8 & No* \\
\hline
\end{tabular}

Table 1 Transcript of the reports of the subset of nine patients who were biopsied at two different times: original (quadriceps) diagnostic biopsy and EDB biopsy. *Patient E's single revertant fibre in the second biopsy represents a negligible increase. Patient I showed traces in the original biopsy and revertants and traces in the second one.

\section{Immunohistochemistry}

Unfixed frozen sections $(6 \mu \mathrm{m})$ were incubated with primary antibodies for $1 \mathrm{~h}$ at room temperature, followed by three washes in PBS, and incubation with biotinylated secondary anti-mouse or anti-rabbit antibodies (Amersham UK, 1:200) for $1 \mathrm{~h}$ at room temperature. Samples were then incubated with streptavidin conjugated to Alexa 594 (Invitrogen UK, 1:1000) for $15 \mathrm{~min}$ at room temperature and washed in PBS before mounting in Histomount (National Diagnostics). All samples were examined and photographed with a Leica epifluorescent microscope.

The antibodies systematically used were: Dys 3 (exon 13) (1:10), Dys 2 (last 17 amino acids of C- terminus) (1:20) [31] and Utrophin (DRP2 1:5). In a subset of samples the following antibodies were additionally used: $\beta$ Dystroglycan (BDG), $\alpha$-sarcoglycan (ASG), spectrin and dystrophin (Dys1, (rod domain), P7 (rod domain, exons 56-60) [32] and Mandys106 (rod domain, exon 43) [33]. All primary antibodies except P7, a rabbit polyclonal antibody [32,34] were monoclonal antibodies from Novocastra Laboratories Ltd except MANDYS106 (gift from Prof. G. Morris, Oswestry).

\section{Study of the biopsy reports}

The same pathologist, CAS, reported all muscle biopsies in an 8-year period and systematically commented on traces and revertants (Supplementary table 1). To validate her analysis, the immunohistochemical analysis in a subset of 10 patients was repeated, analyzed blindly by two other researchers (LF and VA) and compared to the original reports (Supplementary table 2).

When a second biopsy was collected from 9 patients, it was studied and reported by the same pathologist. When studying the incidence of residual dystrophin in our patients, only the original diagnostic reports were included in the analysis, to avoid duplication.

\section{Relative intensity measurements}

New sections from the sixteen biopsies used for quantification were stained and analysed under epifluorescence using a Leica DMR microscope linked to Metamorph (Universal 
Imaging Inc., Downington, PA) as described previously[35]. To summarize, each sample was studied with all eight different antibodies and four images captured per antibody. In all experiments, sections from control and experimental muscles were immunostained simultaneously and, for each antibody except utrophin, used to set the capture settings for the DMD samples. Four images were captured in each section and captured images were analyzed by the Metamorph program, to measure intensities of different areas (Supplementary figure 1). Ten different regions of interests (ROIs), each in a different fibre, were randomly selected per image. These regions included both the cytoplasm and the sarcolemma of fibres, so that a maximum value corresponding to the sarcolemma and a minimum value corresponding to the cytoplasm were used in the analysis. In total, for each experiment, 40 different measurements were taken per antibody per sample. Measurements of the intensity of spectrin from serial sections were used as an internal control to normalise the measurements and these were expressed as a percentage of the control for illustration purposes.

\section{Data analysis}

Statistical analysis was performed with the SPSS package (SPSS Inc, USA). Mann-Whitney analysis was used to compare the groups.

\section{Results}

Revertant fibres are common, but represent a very low percentage of total muscle fibres in DMD muscle.

Biopsy reports were examined for references to residual dystrophin expression. The results were divided into "no revertant fibres and no traces present", "traces of dystrophin only", "revertant fibres only" and "revertant fibres and traces".

Of 63 DMD biopsy reports examined, 47\% presented revertant fibers, $32 \%$ traces and $36 \%$ had neither. $15 \%$ showed both revertant fibres and traces. In the revertant group, 19\% had only one revertant fiber ( $9 \%$ of total samples), with the rest described as having "several" as the only reference to the quantity of revertant fibres. This last group represents $38 \%$ of the total.

\section{No correlation between revertant fibres or traces and Motor Ability Score}

Data on the best MAS and the patient's age before prednisone treatment was initiated were available for 35/63 (55\%) patients. Residual dystrophin was mentioned in the muscle biopsy reports of 18 of these patients, but not in the remaining 17 patients. Both patients' groups demonstrated similar MAS (Figure 1a).

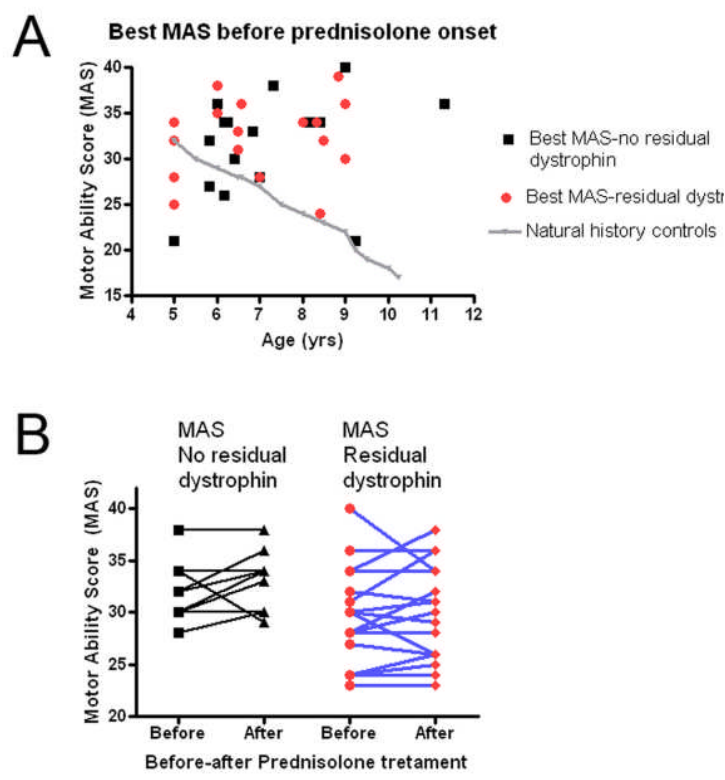

Figure 1 A) Best Motor Ability Score of DMD patients whilst steroid naïve versus natural history data. B) Motor Ability Score of DMD patients with and without dystrophin residual expression revertants at baseline and within 3-6 months of steroid treatment.

Data on the best MAS and the corresponding patient's age at the baseline (before starting steroids) and at the first follow-up (within 3-6 months) of prednisolone treatment were available for $28 / 63$ (44\%) of the patients. Ten patients had no residual dystrophin and were started on prednisolone at $5.68 \pm 1.68$ years and further 18 had residual dystrophin and were started on prednisolone at $6.84 \pm 1.71$ years (age at onset not significantly different). Prednisolone was given as an intermittent 
A

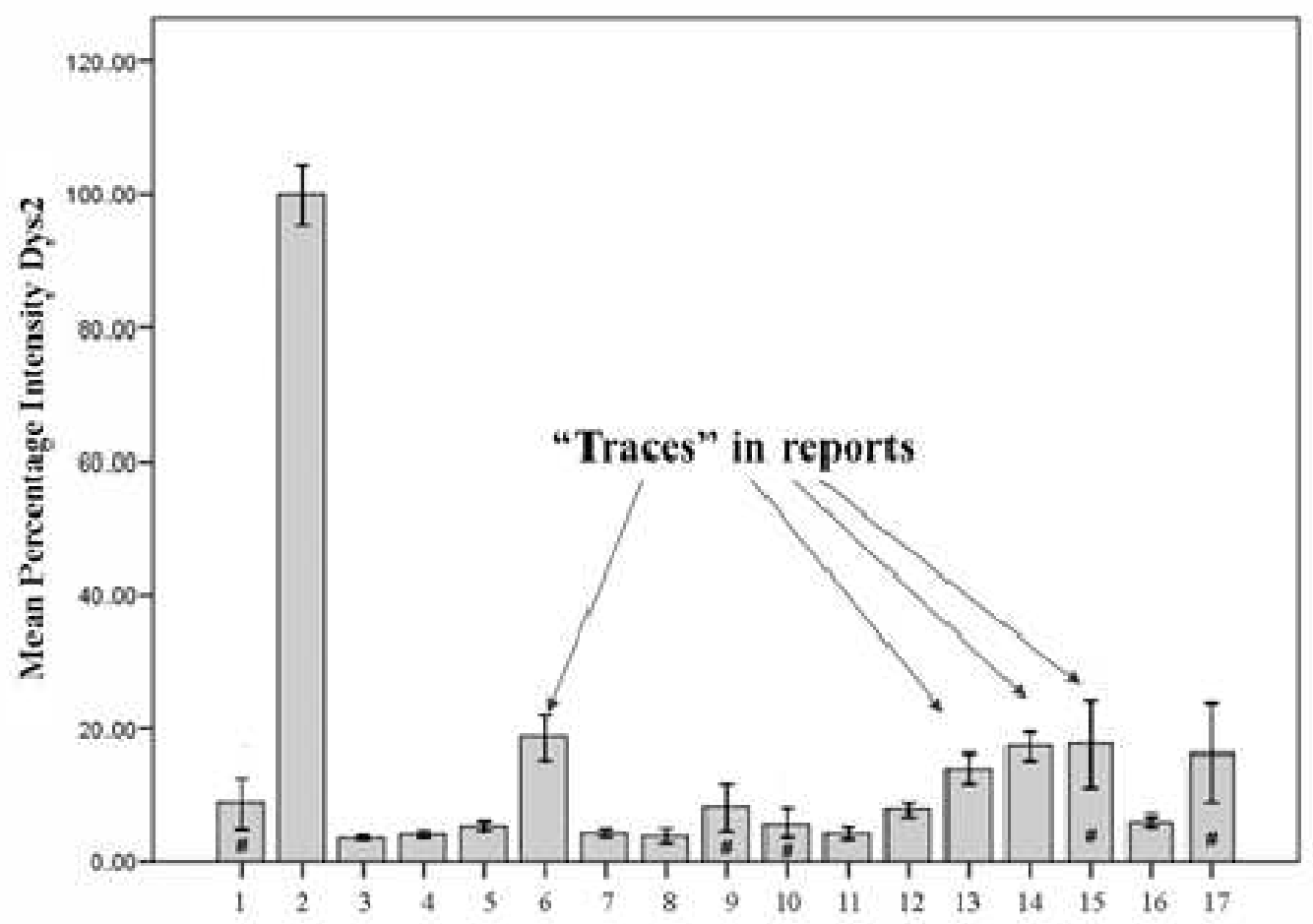

B

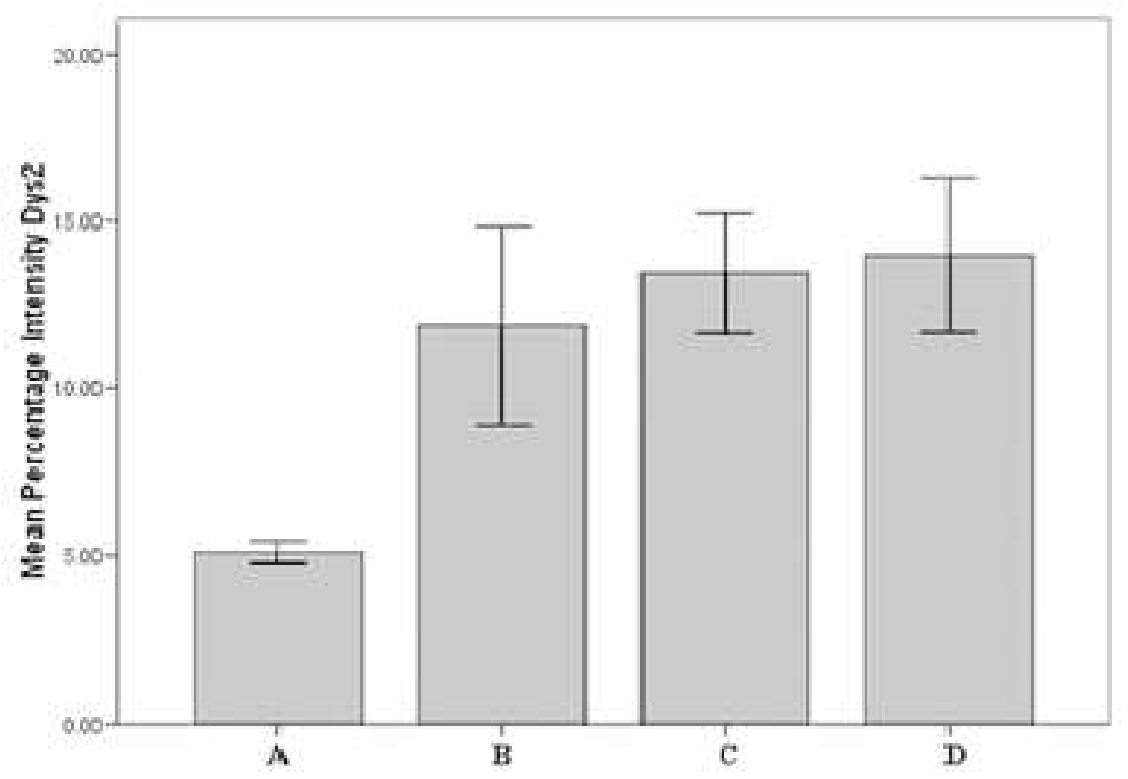

Figure 2 Intensity measurements of images captured from sections of 17 biopsies taken from different patients, after immunostaining with Dys2 were analysed using a semi-quantitative method.

A) The intensity of Dys2 immunostaining is shown as a percentage of the control (2) sample. Arrows show samples that were referred to as containing traces in the reports. Those samples in which revertant fibres were reported are highlighted with the \# symbol. Error bars +/- 2 s.e.m.

B) Sections immunostained with Dys2 showed significant differences $(p<0.001)$ between those that were reported to have neither traces or revertant fibres $(A)$ and those that were reported to have revertant fibres only $(B)$, or traces only $(C)$, or both revertant fibres and traces $(D)$. 
regime using either a regime of 10 days on and 10 days off $(11 / 13$ in the group with no residual dystrophin and $13 / 15$ in the group with residual dystrophin) or a regime of 10 days on and 20 days off $(2 / 13$ in the residual dystrophin group and $2 / 15$ in the residual dystrophin group). The dose was kept at $0.75 \mathrm{mg} / \mathrm{kg} /$ day regardless of the regime. There was no difference in the patients' response to prednisolone at 3-6 months between those who had residual dystrophin or not (Figure $1 b$ ).

The level of trace dystrophin expression can be measured semi-quantitatively using the

\section{Metamorph programme}

The results of the intensity analysis of the biopsies were compared with the references to traces found in their histopathological reports $(n=16)$. Relative quantitative intensity measurements were higher in those samples that had been reported as containing traces than those which reports did not mention traces (Figure 2a). When samples were grouped according to the presence of either revertant fibres or traces, those that had been labeled as containing neither revertant fibres or traces showed significantly less relative intensity than the other groups (one way ANOVA, $p<0.001$ ) (Figure $2 b$ ).
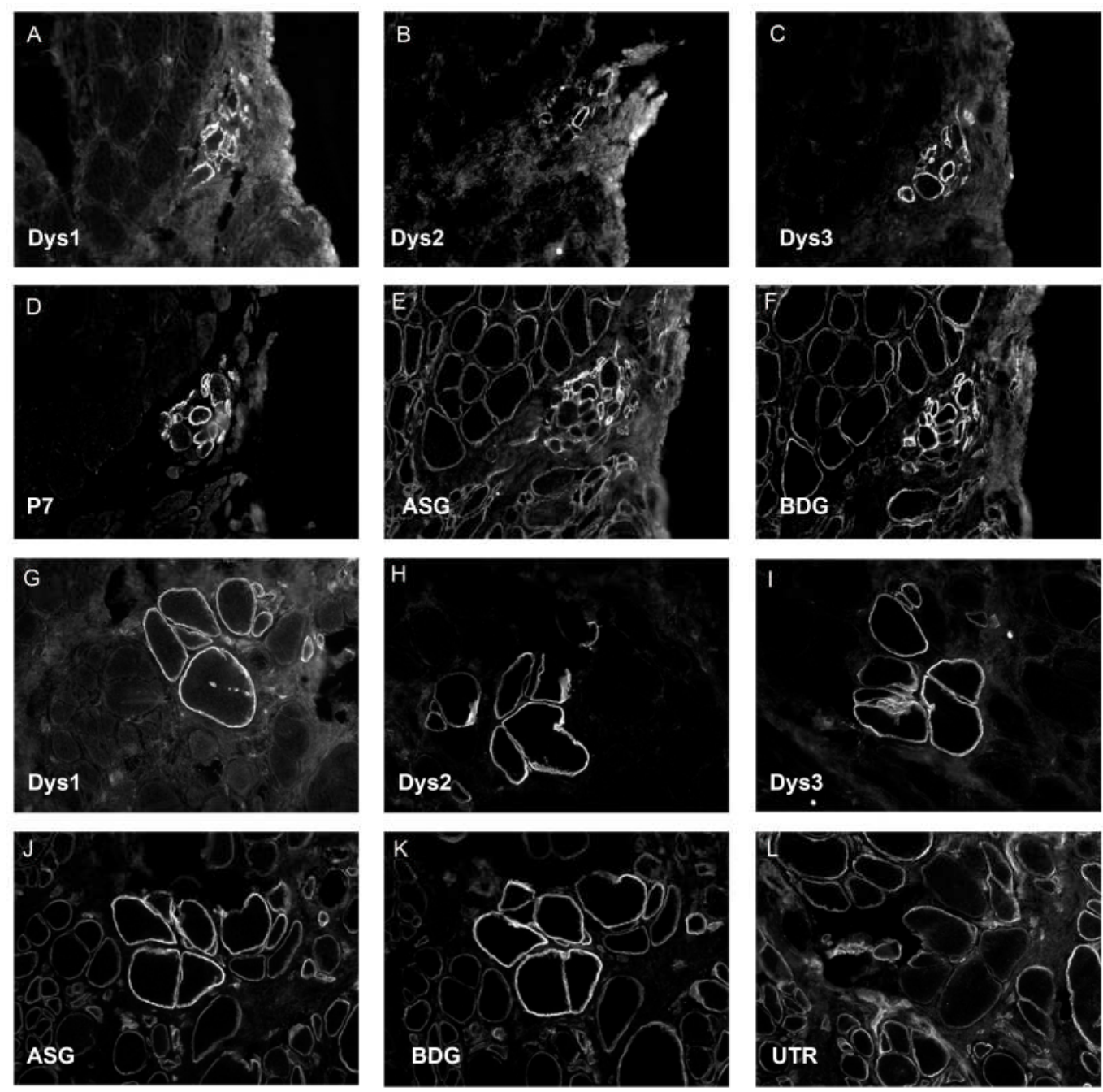

Figure 3 Cryosections from quadriceps muscle biopsies from: muscle from a patient with a deletion in dystrophin exons 46-51 taken at diagnosis (panels A-F) and six years later (panels G-L) stained with antibodies to dystrophin: Dys1 (A and G), Dys2 (B and H), Dys3 ( $C$ and $\mathrm{I}$ ) and P7 (D), alpha-sarcoglycan (E and J), beta-dystroglycan ( $F$ and $K$ ) and utrophin (L). A cluster of revertant, dystrophin-positive fibres is seen in each series of sections. Revertant fibres also contain alpha-sarcoglycan ( $E$ and $J$ ) and beta-dystroglycan ( $F$ and $K$ ). Utrophin is expressed in the dystrophin-negative muscle fibres, but is down-regulated in the revertant fibres (L).

Correspondence to: Jennifer Morgan, The Dubowitz Neuromuscular Centre, $1^{\text {st }}$ Floor, UCL Institute of Child Health, 30 Guilford Street, London, WC1N EH Tel: +44 (0)20 7905 2874, Fax: +44 (0)20 7905 2832;

j.morgan@ich.ucl.ac.uk 
$\boldsymbol{\beta}$-dystroglycan and $\boldsymbol{\alpha}$-sarcoglycan are coexpressed at higher levels in revertant fibers

When analysed by immunohistochemical techniques, revertant fibres were easily identifiable from the fibres surrounding them, with antidystrophin antibodies as they had strong staining with the Dys1, Dys2 and Dys3 antibodies used in the diagnostic panel. In addition revertant fibres also had higher levels of the proteins of the dystrophin associated complex $\beta$-dystroglycan and $\alpha$ - sarcoglycan. The opposite phenomenon was observed in the case of utrophin, which was present at a higher level in fibres surrounding revertant fibres than in the revertant fibres themselves. This was clearly visible in both diagnostic quadriceps and EDB biopsies collected years after diagnosis (Figure 3). Intriguingly, in the muscle biopsy from a different patient with a deletion in exons 45-50 (Patient 65, Supplementary table 1 ), a cluster of revertant fibres that was recognised with Dys 2 and Dys3, but not Dys1 and Mandys106 antibodies, was surrounded by fibres with traces of dystrophin, recognised by Mandys106 antibody, which binds to exon 43, but not the other antibodies (Figure 4). This clearly illustrates that, in this muscle biopsy, different epitopes are expressed by the revertant fibres and the fibres containing dystrophin traces, indicating that the mechanisms involved in dystrophin expression in these two types of fibre can be different.

The number of revertant fibres and amount of traces do not change with time.

Quadriceps muscle biopsies that had been collected at the time of diagnosis were compared with the EDB muscle biopsies obtained several years later (average 7.3 years later). It was observed that those patients whose original biopsy report mentioned revertant fibres or traces presented the same features in the report of the more recent EDB biopsy (Figure 3 and Table 2). In one particular patient, we cut new sections, stained them and compared three muscle samples: the original quadriceps biopsy and both the EDB and the paraspinal muscle biopsies obtained 6.7 years later at the time of spinal fusion. In this patient, the dystrophin expression observed in the quadriceps correlated very closely with the EDB and paraspinal muscle (Patient $\mathrm{G}$ in tables 1 and supplementary table 1).

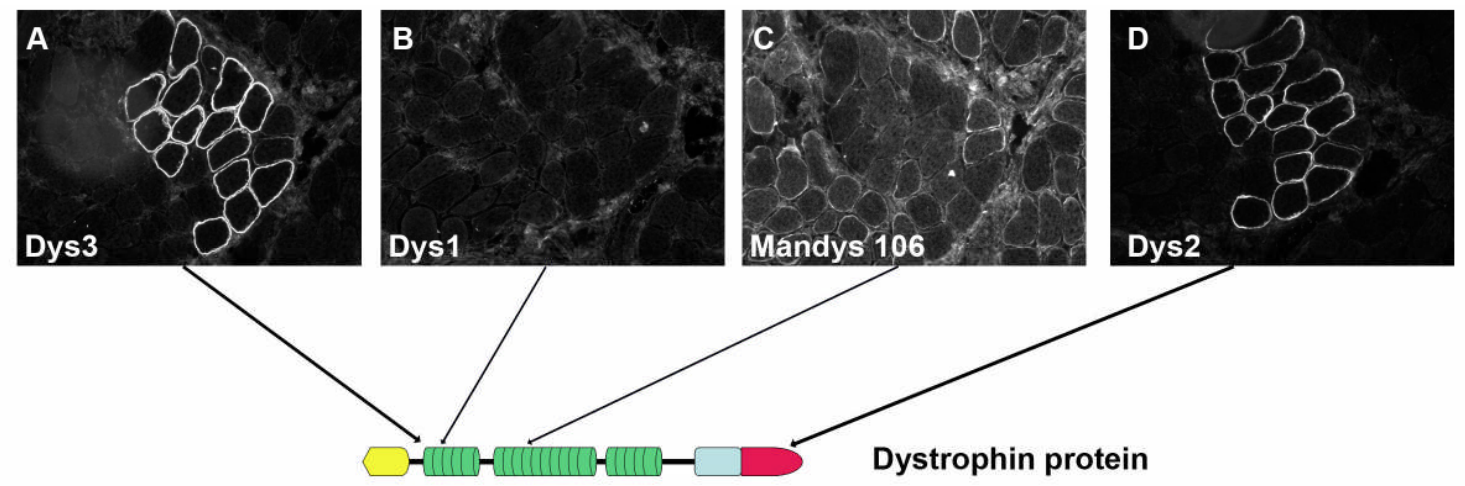

Figure 4 Cryosections from biceps muscle from a DMD patient (patient 65, supplementary table 1, del 45-50) showing a cluster of revertant fibres, clearly visible with anti-dystrophin antibodies Dys2 and Dys3 (A and D) , but not with Dys1 (B). The same area shows dystrophin traces only stained with Mandys 106 antibody, while revertant fibres are not visible with this antibody (C).

\section{Discussion}

Several studies have previously reported the pattern of dystrophin expression in DMD patients; the notion that up to $50 \%$ of them have revertant fibres is fully acknowledged in the literature $[1,5,6,8-10]$. Less emphasis has been placed on the expression of dystrophin traces in DMD. While from a diagnostic perspective revertants and traces do not produce any difficulty, their occurrence could make the interpretation and quantification of efficacy of experimental therapies 
complicated. Indeed a number of different therapeutic strategies aiming to restore dystrophin expression are currently being trialed in DMD: these include i) antisense oligonucleotides which induce exon skipping in DMD patients with out-of-frame deletions; ii) PTC124, which is thought to enable the translation machinery to bypass nonsense mutations; iii) adeno-associated viruses coding for mini-dystrophins; iv) plasmid DNA injection of dystrophin [36]. In these early studies, restoration of dystrophin expression following therapy might be relatively limited, especially in boys receiving low doses of medicinal product in dose escalation studies. The possibility that traces of dystrophin or revertant fibres may be present in different percentages in different muscles of the body, or that this expression could increase with age [19], also needs to be considered. Our study was therefore aimed to provide more information on these aspects, by studying the prevalence of revertant fibres and traces in a large population of DMD patients. We found revertant fibres in $47 \%$ of the patients, which corresponds with figures reported in previous studies. When traces were also considered, residual dystrophin was found in $64 \%$ of the patients studied. $15 \%$ of biopsies studied had both strongly-staining revertant fibres and fibres containing unequivocal, but less intense, dystrophin staining (traces), showing that both types of fibres may, but not necessarily, be present in the same muscle.

These dystrophins cannot be distinguished from each other by immunoblotting; in addition, western blots may not be sensitive enough to detect very small amounts of dystrophin produced in either revertant fibres or traces, nor quantitative enough at the high and low end of the signal [38]. Western blotting requires more muscle than immunostaining and there was insufficient muscle from several patients to perform this analysis. Despite limitations also of this technique, immunostaining has been used in many studies in a semi-quantitative manner $[25,26]$; in addition the number of dystrophin positive fibres in a representative section of muscle has been shown to correlate with the amount of dystrophin on a western blot [37]. A problem with both western blotting and immunostaining is sensitivity and specificity of the antibodies used. Those we have used in this study are very specific and are widelyused in routine diagnosis [3,38], but some are clearly more sensitive than others [25]. The problem is compounded in studies of revertant fibres, which express lower than normal levels of dystrophin [35] and only express some epitopes of the protein [19]. After consideration of all advantages and drawbacks of immunostaining and western blotting, we chose to use immunostaining, rather than western blotting, for our study.

Studies in the $m d x$ mouse suggest that revertant fibres could increase in number with age [19] and anecdotal evidence was found in some human studies showing a higher percentage of revertant fibres in older patients $[8,13]$. This could be relevant as it might confuse the analysis of dystrophin restoration in treated patients. Fanin et al. (1992)[8] found more than $1 \%$ dystrophinpositive fibres only in DMD patients over 6 years of age and the same group (1995) [13] reported that older patients had more revertant fibres per cluster and that there was a direct correlation between the age of the patient and prevalence of the percentage of total fibres that were dystrophin positive. However a limitation of that study, performed in 49 individuals, is that the majority of the biopsies were from patients under 10 years of age and the lack of longitudinal data on the same patients. In addition, this study reported the percentage, not the number, of dystrophin positive fibres. If the presence of dystrophin confers a survival advantage to the fibre, dystrophin negative fibres will be lost, whereas dystrophin positive fibres will survive, which may lead to an increase in the percentage, but not the total number, of dystrophin positive fibres with time.

In our study, we compared quadriceps biopsies with EDB biopsies performed in the 
same patients 6-10 years later and find no obvious increase in numbers of dystrophin positive fibres, nor did we find larger clusters of revertant fibres at the later timepoint. Those patients who had no dystrophin in the original biopsy did not have any in the second biopsy and vice versa.

The apparent lack of expansion of revertant clusters in human DMD muscle may be due to either lack of functional muscle stem cells, or to the environment within DMD muscle being sub-optimal for stem cell function. In mice, expansion of revertant fibres, which appears to be a clonal event, is dependent on muscle regeneration [39] and does not occur under conditions in which muscle stem cells are either ablated or not activated [40].

There is ongoing discussion about the possibility that dystrophin-positive fibres may offer a biological advantage by lessening the severity of the disease course in DMD $[13,17,41]$. We therefore correlated dystrophin expression both with the functional abilities of patients as assessed by the MAS, whilst steroid naïve, and with response to corticosteroid treatment. Although our results should be interpreted with caution, as only $55 \%$ of patients had detailed assessments at the peak of their motor ability whilst steroid naïve, our data do not indicate a difference in function between patients with or without residual dystrophin expression. Different explanations could account for these findings. One possibility is that the dystrophin produced in these fibres, although correctly localised, is lacking crucial binding domains, and is therefore not able to efficiently protect the fibre from damage. Indeed, internally deleted dystrophins lacking an appreciable portion of the rod domain are ineffective in reducing myofibre necrosis and restoring muscle function in transgenic $m d x$ mice [42-45]. Another possibility is that this phenomenon occurs in so few fibres that it is not sufficient to be beneficial to properties of the entire muscle. It is quite likely that the amount of residual dystrophin had not reached the threshold level of protein necessary to effect a functional improvement in these patients [46].

In previous studies, DMD muscles containing traces and revertant fibres have been analysed by western blotting and shown to contain higher amounts of dystrophin than muscles without either, but traces or revertant fibres could not be studied separately [17]. We have shown that the presence of traces can be identified not only by visual inspection of the muscle biopsy slides but also by a semi-quantitative capturing method, that reveals dystrophin protein to be significantly higher in fibres with dystrophin traces compared to the neighboring ones $[25,26]$. The mechanism responsible for the production of dystrophin traces may well be different from revertant fibres, but in both cases, dystrophin is correctly localized at the sarcolemma. The molecular basis of trace dystrophin expression remains to be elucidated, but we show that, in a biopsy of a patient with a deletion in exons 45-50, a cluster of revertant fibres expresses different dystrophin epitopes than the surrounding fibres that contain traces of dystrophin. This suggests that the dystrophin proteins produced in this group of fibres may have been produced by different mechanisms in this patient.

One limitation of our study is that we have not been able to study the same muscle analysed in the original diagnostic biopsy, but compared dystrophin expression of this muscle with the EDB muscle. While we cannot exclude the possibility that a repeated quadriceps biopsy might have shown a different percentage of fibres with residual expression, the very close correlation between the presence of dystrophin in the two biopsies (and also a third paraspinal muscle biopsy in a single patient) is very encouraging and makes this possibility unlikely. In addition, the histological analysis of the EDB muscle showed a clear advanced dystrophic pathology in all patients, 
suggesting that the degeneration and regenerative process does occur indeed in this muscle as well [25].

We conclude that dystrophin expression, either in revertant fibres, or in fibres containing trace amounts of dystrophin, is common phenomenon in DMD patients but, in contrast to the $m d x$ mouse, we found no evidence of clonal expansion of revertant fibres over time, at least when comparing different muscles. This lack of increase in number of dystrophin-containing fibres with age will facilitate the interpretation of strategies designed to restore dystrophin expression and facilitates the recruitment of patients into experimental therapies, provided the patient had already had a diagnostic biopsy.

\section{References}

1. Hoffman EP, Brown RH, Jr., Kunkel LM: Dystrophin: the protein product of the Duchenne muscular dystrophy locus. Cell 1987, 51:919-928.

2. Koenig $M$, Hoffman EP, Bertelson $C J$, Monaco AP, Feener $C$, Kunkel LM: Complete cloning of the Duchenne muscular dystrophy (DMD) CDNA and preliminary genomic organization of the DMD gene in normal and affected individuals. Cell 1987, 50:509-517.

3. Muntoni F: Is a muscle biopsy in Duchenne dystrophy really necessary? Neurology 2001, 57:574-575.

4. Hoffman EP, Morgan JE, Watkins SC, Partridge TA: Somatic reversion/suppression of the mouse mdx phenotype in vivo. I Neurol Sci 1990, 99:9-25.

5. Nicholson LV, Davison K, Johnson MA, Slater CR, Young C, Bhattacharya S, Gardner-Medwin D, Harris JB: Dystrophin in skeletal muscle. II. Immunoreactivity in patients with Xp21 muscular dystrophy. I Neurol Sci 1989, 94:137146.

6. Nicholson LV, Johnson MA, Gardner-Medwin $D$, Bhattacharya S, Harris JB: Heterogeneity of dystrophin expression in patients with Duchenne and Becker muscular dystrophy. Acta Neuropathol 1990, 80:239-250.

7. Kissel JT, Burrow KL, Rammohan KW, Mendell JR: Mononuclear cell analysis of muscle biopsies in prednisone-treated and untreated Duchenne muscular dystrophy. CIDD Study Group. Neurology 1991, 41:667-672.

8. Fanin $M$, Danieli GA, Vitiello L, Senter L, Angelini C: Prevalence of dystrophin-positive fibers in 85 Duchenne muscular dystrophy patients. Neuromuscul Disord 1992, 2:41-45.

9. Klein CJ, Coovert DD, Bulman DE, Ray PN, Mendell JR, Burghes AH: Somatic reversion/suppression in

\section{Acknowledgements.}

This work was funded by the Department of Health (UK). The Authors wish to thank the members of the UK Muscular Dystrophy Experimental treatment (MDEX, http://www.mdex.org.uk/index.php) consortium for helpful discussion (Prof D. Wells; Dr. M. Wood; Dr Ian Graham and Prof George Dickson) and the Muscular Dystrophy Campaign, Action Duchenne, DMD support group and participating patients for the support to our study. We thank Prof. Glenn Morris, Oswestry and the MDA Monoclonal Antibody Resource for MANDSY106. JEM was funded by an MRC joint collaborative career development award in stem cell research and by a Wellcome Trust university award. The Biobank of the MRC Centre for Neuromuscular Diseases (CNMD) and the TREAT-NMD initiative are also gratefully acknowledged.

Duchenne muscular dystrophy (DMD): evidence supporting a frame-restoring mechanism in rare dystrophin-positive fibers. Am J Hum Genet 1992, 50:950-959.

10. Sherratt TG, Vulliamy T, Dubowitz V, Sewry CA, Strong $\mathrm{PN}$ : Exon skipping and translation in patients with frameshift deletions in the dystrophin gene. Am J Hum Genet 1993, 53:1007-1015.

11. Thanh LT, Nguyen TM, Helliwell TR, Morris GE: Characterization of revertant muscle fibers in Duchenne muscular dystrophy, using exonspecific monoclonal antibodies against dystrophin. Am J Hum Genet 1995, 56:725$\underline{731 .}$

12. Schatzberg SJ, Anderson LV, Wilton SD, Kornegay JN, Mann CJ, Solomon GG, Sharp NJ: Alternative dystrophin gene transcripts in golden retriever muscular dystrophy. Muscle Nerve 1998, 21:991-998.

13. Fanin M, Danieli GA, Cadaldini M, Miorin M, Vitiello L, Angelini C: Dystrophin-positive fibers in Duchenne dystrophy: origin and correlation to clinical course. Muscle Nerve 1995, 18:1115-1120.

14. Uchino $M$, Tokunaga $M$, Mita $S$, Uyama $E$, Ando $Y$, Teramoto $H$, Miike $T$, Ando $M$ : PCR and immunocytochemical analyses of dystrophinpositive fibers in Duchenne muscular dystrophy. J Neurol Sci 1995, 129:44-50.

15. Winnard AV, Mendell JR, Prior TW, Florence J, Burghes AH: Frameshift deletions of exons 3-7 and revertant fibers in Duchenne muscular dystrophy: mechanisms of dystrophin production. Am J Hum Genet 1995, 56:158166.

16. Nicholson LV: The "rescue" of dystrophin synthesis in boys with Duchenne muscular dystrophy. Neuromuscul Disord 1993, 3:525-531. 
17. Nicholson LV, Johnson MA, Bushby KM, GardnerMedwin D: Functional significance of dystrophin positive fibres in Duchenne muscular dystrophy. Arch Dis Child 1993, 68:632-636.

18. Danko I, Chapman V, Wolff JA: The frequency of revertants in $\mathrm{mdx}$ mouse genetic models for Duchenne muscular dystrophy. Pediatr Res 1992, 32:128-131.

19. Lu QL, Morris GE, Wilton SD, Ly T, Artem'yeva OV, Strong $P$, Partridge TA: Massive idiosyncratic exon skipping corrects the nonsense mutation in dystrophic mouse muscle and produces functional revertant fibers by clonal expansion. J Cell Biol 2000, 148:985-996.

20. Sironi M, Cagliani R, Pozzoli U, Bardoni A, Comi GP, Giorda R, Bresolin N: The dystrophin gene is alternatively spliced throughout its coding sequence. FEBS Lett 2002, 517:163-166.

21. Matsumura $K$, Tome $F M$, Collin $H$, Leturca $F$, Jeanpierre M, Kaplan JC, Fardeau M, Campbell KP: Expression of dystrophin-associated proteins in dystrophin-positive muscle fibers (revertants) in Duchenne muscular dystrophy. Neuromuscul Disord 1994, 4:115-120.

22. Wallgren-Pettersson C, Jasani B, Rosser LG, Lazarou LP, Nicholson LV, Clarke A: Immunohistological evidence for second or somatic mutations as the underlying cause of dystrophin expression by isolated fibres in Xp21 muscular dystrophy of Duchenne-type severity. I Neurol Sci 1993, 118:56-63.

23. Fall AM, Johnsen $R$, Honeyman $K$, Iversen $P$, Fletcher $S$, Wilton SD: Induction of revertant fibres in the $\mathrm{mdx}$ mouse using antisense oligonucleotides. Genet Vaccines Ther 2006, 4:3.

24. Bertoni C, Lau C, Rando TA: Restoration of dystrophin expression in $\mathrm{mdx}$ muscle cells by chimeraplast-mediated exon skipping. Hum Mol Genet 2003, 12:1087-1099.

25. Kinali $M$, Arechavala-Gomeza $V$, Feng L, Cirak S, Hunt D, Adkin C, Guglieri $M$, Ashton $E$, Abbs $S$, Nihoyannopoulos $P$, et al.: Local restoration of dystrophin expression with the morpholino oligomer AVI-4658 in Duchenne muscular dystrophy: a single-blind, placebo-controlled, dose-escalation, proof-of-concept study. Lancet Neurol 2009, 8:918-928.

26. van Deutekom JC, Janson AA, Ginjaar IB, Frankhuizen WS, Aartsma-Rus A, Bremmer-Bout $M$, den Dunnen JT, Koop K, van der Kooi AJ, Goemans $\mathrm{NM}$, et al.: Local dystrophin restoration with antisense oligonucleotide PRO051. N Engl J Med 2007, 357:2677-2686.

27. Hirawat $S$, Welch EM, Elfring GL, Northcutt VJ, Paushkin S, Hwang S, Leonard EM, Almstead NG, Ju W, Peltz SW, et al.: Safety, tolerability, and pharmacokinetics of PTC124, a nonaminoglycoside nonsense mutation suppressor, following single- and multipledose administration to healthy male and female adult volunteers. I Clin Pharmacol 2007, 47:430-444.

28. Emery A, Muntoni F: Duchenne muscular dystrophy. Oxford University Press

2003:pp 280.

29. Scott OM, Hyde SA, Goddard C, Dubowitz V: Quantitation of muscle function in children: a prospective study in Duchenne muscular dystrophy. Muscle Nerve 1982, 5:291-301.

30. Manzur AY, Kuntzer T, Pike M, Swan A: Glucocorticoid corticosteroids for Duchenne muscular dystrophy. Cochrane Database Syst Rev 2004:CD003725.

31. Ervasti JM, Ohlendieck K, Kahl SD, Gaver MG, Campbell KP: Deficiency of a glycoprotein component of the dystrophin complex in dystrophic muscle. Nature 1990, 345:315-319.

32. Lu QL, Rabinowitz A, Chen YC, Yokota T, Yin H, Alter J, Jadoon A, Bou-Gharios G, Partridge T: Systemic delivery of antisense oligoribonucleotide restores dystrophin expression in body-wide skeletal muscles. Proc Natl Acad Sci U S A 2005, 102:198-203.

33. Nguyen TM, Morris GE: Use of epitope libraries to identify exon-specific monoclonal antibodies for characterization of altered dystrophins in muscular dystrophy. Am J Hum Genet 1993, 52:1057-1066.

34. Sherratt TG, Vulliamy T, Strong PN: Evolutionary conservation of the dystrophin central rod domain. Biochem J 1992, 287 ( Pt 3):755-759.

35. Arechavala-Gomeza $V$, Kinali $M$, Feng $L$, Brown $S C$, Sewry C, Morgan JE, Muntoni F: Immunohistological intensity measurements as a tool to assess sarcolemma- associated protein expression. Neuropathol Appl Neurobiol 2009.

36. Romero NB, Braun $S$, Benveniste $O$, Leturcq $F$, Hogrel JY, Morris GE, Barois A, Eymard B, Payan C, Ortega $V$, et al.: Phase I study of dystrophin plasmid-based gene therapy in Duchenne/Becker muscular dystrophy. Hum Gene Ther 2004, 15:1065-1076.

37. Nicholson LV, Johnson MA, Bushby KM, GardnerMedwin D, Curtis A, Ginjaar IB, den Dunnen JT, Welch JL, Butler TJ, Bakker E, et al.: Integrated study of 100 patients with Xp21 linked muscular dystrophy using clinical, genetic, immunochemical, and histopathological data. Part 2. Correlations within individual patients. J Med Genet 1993, 30:737-744.

38. Dubowitz V, Sewry C: Muscle Biopsy: A practical approach edn Third Edition. Edited by Houston MJ, Cook L: SAUNDERS Elsevier; 2007.

39. Crawford GE, LU QL, Partridge TA, Chamberlain JS: Suppression of revertant fibers in $\mathrm{mdx}$ mice by expression of a functional dystrophin. Hum Mol Genet 2001, 10:2745-2750.

40. Yokota T, Lu QL, Morgan JE, Davies KE, Fisher R, Takeda S, Partridge TA: Expansion of revertant fibers in dystrophic $\mathrm{mdx}$ muscles reflects activity of muscle precursor cells and serves 
as an index of muscle regeneration. J Cell Sci 2006, 119:2679-2687.

41. Nicholson LV, Bushby KM, Johnson MA, GardnerMedwin D, Ginjaar IB: Dystrophin expression in Duchenne patients with "in-frame" gene deletions. Neuropediatrics 1993, 24:93-97.

42. Rafael JA, Cox GA, Corrado K, Jung D, Campbell KP, Chamberlain JS: Forced expression of dystrophin deletion constructs reveals structure-function correlations. I Cell Biol 1996, 134:93-102.

43. Rafael JA, Sunada Y, Cole NM, Campbell KP, Faulkner JA, Chamberlain JS: Prevention of dystrophic pathology in $\mathrm{mdx}$ mice by a truncated dystrophin isoform. Hum Mol Genet 1994, 3:1725-1733.
44. Phelps SF, Hauser MA, Cole NM, Rafael JA, Hinkle RT, Faulkner JA, Chamberlain JS: Expression of fulllength and truncated dystrophin mini-genes in transgenic mdx mice. Hum Mol Genet 1995, 4:1251-1258.

45. Corrado K, Rafael JA, Mills PL, Cole NM, Faulkner JA, Wang K, Chamberlain JS: Transgenic mdx mice expressing dystrophin with a deletion in the actin-binding domain display a "mild Becker" phenotype. J Cell Biol 1996, 134:873-884.

46. Neri M, Torelli S, Brown S, Ugo I, Sabatelli $P$, Merlini L, Spitali P, Rimessi P, Gualandi F, Sewry C, et al.: Dystrophin levels as low as $\mathbf{3 0 \%}$ are sufficient to avoid muscular dystrophy in the human. Neuromuscul Disord 2007, 17:913-918. 